\title{
IGUSA'S CONJECTURE FOR EXPONENTIAL SUMS: OPTIMAL ESTIMATES FOR NONRATIONAL SINGULARITIES
}

\author{
RAF CLUCKERS ${ }^{\circledR 1,2}$, MIRCEA MUSTAŢA $\breve{A}^{3}$ and KIEN HUU NGUYEN ${ }^{\circledR 4}$ \\ ${ }^{1}$ CNRS, Univ. Lille, UMR 8524 - Laboratoire Paul Painlevé, F-59000 Lille, France \\ ${ }^{2}$ KU Leuven, Department of Mathematics, B-3001 Leuven, Belgium; \\ email: Raf.Cluckers@univ-lille.fr \\ ${ }^{3}$ Department of Mathematics, University of Michigan, Ann Arbor, MI 48109, USA; \\ email: mmustata@umich.edu \\ ${ }^{4}$ KU Leuven, Department of Mathematics, Celestijnenlaan 200B, B-3001 Leuven, Belgium; \\ email: kien.nguyenhuu@kuleuven.be
}

Received 26 October 2019; accepted 1 May 2019

\begin{abstract}
We prove an upper bound on the log canonical threshold of a hypersurface that satisfies a certain power condition and use it to prove several generalizations of Igusa's conjecture on exponential sums, with the log canonical threshold in the exponent of the estimates. We show that this covers optimally all situations of the conjectures for nonrational singularities by comparing the $\log$ canonical threshold with a local notion of the motivic oscillation index.
\end{abstract}

2010 Mathematics Subject Classification: 11L07, 11S40 (primary); 11L05, 14E30 (secondary)

\section{Introduction}

Igusa's conjecture on exponential sums predicts upper bounds for $\left|S_{f}(a)\right|$ in terms of $a$, where $f$ is a nonconstant polynomial over $\mathbb{Z}$ in $n$ variables, $a$ runs over the positive integers, and $S_{f}(a)$ is the finite exponential sum

$$
S_{f}(a):=\frac{1}{a^{n}} \sum_{x \in(\mathbb{Z} / a \mathbb{Z})^{n}} \exp \left(\frac{2 \pi i f(x)}{a}\right) .
$$

(C) The Author(s) 2019. This is an Open Access article, distributed under the terms of the Creative Commons AttributionNonCommercial-ShareAlike licence (http://creativecommons.org/licenses/by-nc-sa/4.0/), which permits non-commercial re-use, distribution, and reproduction in any medium, provided the same Creative Commons licence is included and the original work is properly cited. The written permission of Cambridge University Press must be obtained for commercial re-use. 
If $a$ runs only over the positive powers $p^{m}$ of a fixed prime number $p$, these bounds are well known and proved by Igusa; the key point of his conjecture is about varying the prime $p$, as follows. Suppose that for some real $\sigma>0$ and for each prime $p$, there exists a constant $c_{p}>0$ such that

$$
\left|S_{f}\left(p^{m}\right)\right|<c_{p} p^{-m \sigma} \quad \text { for each integer } m \geqslant 2 .
$$

Then the question is generally whether one can take $c_{p}$ independently of the prime $p$ (but still depending on $\sigma$ and $f$ ). In a more explicit form, Igusa puts forward precise values for $\sigma$, the infimum of which relates to the log canonical threshold of $f$ in the case of nonrational singularities and, more generally, to the motivic oscillation index (see below). Conditions on $f$ that were originally imposed by Igusa [26, pages 2 and 170] (like the homogeneity of $f$ and bounds on $\sigma$ ) have been relaxed in several later variants of his question $[4,8,10,15]$.

In this paper, we prove these later variants with the log canonical threshold playing a key role both in the exponent of the upper bounds and in the proofs. The appearance of the log canonical threshold in the exponent of the upper bounds is optimal in many cases: it is only when the hypersurfaces defined by $f-c$, for $c \in \mathbb{C}$, have at worst rational singularities that there is room for an improved exponent; see Section 3.4.

We derive the bounds for the exponential sums from an upper bound on the $\log$ canonical threshold of the hypersurface defined by $f$ in the presence of a certain power condition. This is described in terms of a log resolution for the hypersurface. We prove this upper bound by making use of a finiteness result concerning certain divisorial valuations, a result which follows from the recent progress in the Minimal Model Program [2]. Deriving Igusa's conjecture from the log canonical threshold bound relies on several subtle results on Igusa's local zeta functions, most of which can be found in the overview paper [13] by Denef. These allow us to reduce to finite field exponential sums with multiplicative characters that can be bounded in a way matching our bounds on the log canonical threshold.

We mention that while Igusa's conjectured upper bounds are very natural, his motivation came from their role in obtaining adèlic integrability properties, which in turn were crucial for proving Poisson summation formulas throughout his work [19, 20, 22-26]. These Poisson summation formulas are a step in Igusa's program toward new local-global principles; see [24, page 240].

1.1. The log canonical threshold bound. We begin by stating our result on $\log$ canonical thresholds. Let $X$ be a smooth complex algebraic variety and $D$ a nonempty hypersurface in $X$ defined by $f \in \mathcal{O}_{X}(X)$. We consider a log 
resolution $h: Y \rightarrow X$ of the pair $(X, D)$, which is an isomorphism over $X \backslash D$. Therefore, $h$ is a projective morphism, $Y$ is smooth, and $h^{*}(D)$ is a divisor with simple normal crossings. Note that by our assumption on $h$, the relative canonical divisor $K_{Y / X}$ is supported on $h^{*}(D)_{\text {red }}$.

We write

$$
h^{*}(D)=\sum_{i=1}^{N} N_{i} E_{i} \quad \text { and } \quad K_{Y / X}=\sum_{i=1}^{N}\left(v_{i}-1\right) E_{i},
$$

for positive integers $N_{i}, v_{i}$, and prime divisors $E_{i}$ so that the $\log$ canonical threshold $\operatorname{lct}(f)$ of $f$ is given by

$$
\operatorname{lct}(f)=\min _{i} \frac{v_{i}}{N_{i}}
$$

(see, for example, [35] for an introduction to log canonical thresholds). For every subset $I \subseteq\{1, \ldots, N\}$, we put

$$
E_{I}=\bigcap_{i \in I} E_{i} \quad \text { and } \quad E_{I}^{\circ}=E_{I} \backslash \bigcup_{i \notin I} E_{i},
$$

where $E_{\emptyset}=Y$. By construction, given a (possibly nonclosed) point $P \in Y$, there is a unique $I \subseteq\{1, \ldots, N\}$ such that $P \in E_{I}^{\circ}$; moreover, there is an algebraic system of coordinates $x_{1}, \ldots, x_{n}$ in a neighborhood $X_{0}$ of $P$ such that after relabeling so that $I=\{1, \ldots, m\}$, we have $E_{i} \cap X_{0}=V\left(x_{i}\right)$ if and only if $i \leqslant m$, and we can write

$$
\left.f \circ h\right|_{X_{0}}=u \cdot \prod_{i=1}^{m} x_{i}^{N_{i}},
$$

where $u \in \mathcal{O}_{Y}\left(X_{0}\right)$ is an invertible regular function on $X_{0}$.

For a closed subset (or closed subscheme) $Z$ of $X$, having nonempty intersection with $D$, we denote by $\operatorname{lct}_{Z}(f)$ the largest $\log$ canonical threshold $\operatorname{lct}\left(\left.f\right|_{V}\right)$, where $V$ is an open neighborhood of $Z$. Note that we have $\operatorname{lct}_{Z}(f)=\min _{x \in Z \cap D} \operatorname{lct}_{x}(f)$.

THEOREM 1.1. Suppose that there is a nonempty subset $I \subseteq\{1, \ldots, N\}$ and an open subset $X_{0}$ as above such that $X_{0} \cap E_{I}^{\circ}$ is nonempty and

$$
\left.u\right|_{X_{0} \cap E_{I}^{\circ}}=g^{d}
$$

for some integer $d>1$ with $d \mid N_{i}$ for all $i \in I$ and some $g \in \mathcal{O}\left(X_{0} \cap E_{I}^{\circ}\right)$. In this case, we have the following upper bound for the log canonical threshold of $f$ :

$$
\operatorname{lct}(f) \leqslant \frac{1}{d}+\sum_{i \in I} N_{i}\left(\frac{v_{i}}{N_{i}}-\operatorname{lct}(f)\right) .
$$


More generally, if $\overline{h\left(X_{0} \cap E_{I}^{\circ}\right)} \cap Z \neq \emptyset$, then we have

$$
\operatorname{lct}_{Z}(f) \leqslant \frac{1}{d}+\sum_{i \in I} N_{i}\left(\frac{v_{i}}{N_{i}}-\operatorname{lct}_{Z}(f)\right) .
$$

REMARK 1.2. A slightly more restrictive form of the condition in (1.4) of Theorem 1.1 is formalized and coined as 'power condition' in Section 2.1. Note that the requirement $d \mid N_{i}$ for all $i \in I$ in the theorem makes the condition (1.4) independent of the choice of local coordinates $x_{1}, \ldots, x_{n}$.

1.2. Estimates for exponential sums. Let us now formulate our main results on exponential sums. We work over a ring of integers $\mathcal{O}$ instead of over $\mathbb{Z}$.

Let $f$ be a nonconstant polynomial in $\mathcal{O}[x]$ in $n$ variables $x=\left(x_{1}, \ldots, x_{n}\right)$. Let $Z$ be a closed subscheme of $\mathbb{A}_{\mathcal{O}}^{n}$. Let $L$ be a local field over the ring $\mathcal{O}$, that is, a finite field extension of $\mathbb{Q}_{p}$ or of $\mathbb{F}_{p}((t))$ for some prime number $p$ such that, moreover, there is a unit-preserving ring homomorphism $\mathcal{O} \rightarrow L$. We denote by $\mathcal{O}_{L}$ the valuation ring of $L$ and by $|d x|$ the Haar measure on $L^{n}$, normalized so that $\mathcal{O}_{L}^{n}$ has measure 1 . The number of elements in the residue field $k_{L}$ of $L$ is $q_{L}$ and equals a power of a prime number $p_{L}$. Let $\psi: L \rightarrow \mathbb{C}^{\times}$be a nontrivial additive character on $L$, that is, a (nontrivial) continuous group homomorphism from the additive group of $L$ to $\mathbb{C}^{\times}$. For such data, consider the integral

$$
E_{f, L, \psi}^{Z}:=\int_{\left\{x \in \mathcal{O}_{L}^{n} \mid \bar{x} \in Z\left(k_{L}\right)\right\}} \psi(f(x))|d x|,
$$

where $\bar{x}$ stands for the image of $x$ under the natural projection $\mathcal{O}_{L}^{n} \rightarrow k_{L}^{n}$. We write $E_{f, L, \psi}$ for $E_{f, L, \psi}^{Z}$ when $Z=\mathbb{A}_{\mathcal{O}}^{n}$. Note that the integrals $E_{f, L, \psi}^{Z}$ are in fact finite exponential sums which include the above sums $S_{f}\left(p^{m}\right)$, with $p$ a prime number, as special cases. Moreover, estimating $S_{f}\left(p^{m}\right)$ is the key to estimating $S_{f}(a)$ for general $a$ by the Chinese remainder theorem. In what follows, we write $\operatorname{lct}_{Z}(f)$ for $\operatorname{lct}_{Z_{\mathbb{C}}}(f)$.

DEFINITION 1.3. For a nontrivial additive character $\psi$ on $L$, let $m_{\psi}$ be the unique integer $m$ such that $\psi$ is trivial on $\varpi_{L}^{m} \mathcal{O}_{L}$ and nontrivial on $\varpi_{L}^{m-1} \mathcal{O}_{L}$, where $\varpi_{L}$ is a uniformizer of $\mathcal{O}_{L}$.

Note that for any additive character $\psi$ on $L$ with $m_{\psi}=0$ and any $z \in L^{\times}$, the character $\psi_{z}$ sending $x \in L$ to $\psi(z x)$ satisfies

$$
m_{\psi_{z}}=-\operatorname{ord}(z)
$$


and that all nontrivial additive characters on $L$ are of the form $\psi_{z}$ for varying $z$; see, for example, [27].

Definition 1.4. For a nonconstant polynomial $f$ with coefficients in $\mathbb{C}$ and any subset $Z$ of $\mathbb{C}^{n}$, let

$$
\sigma_{Z}(f)=\min \left\{\operatorname{lct}_{x}(f-b) \mid x \in Z, b=f(x)\right\} .
$$

If $Z=\mathbb{C}^{n}$, then we simply write $\sigma(f)$ for $\sigma_{Z}(f)$. Given a closed subscheme $Y$ of $\mathbb{A}_{\mathcal{O}}^{n}$ or of $\mathbb{A}_{\mathbb{C}}^{n}$, we write $\sigma_{Y}(f)$ for $\sigma_{Y(\mathbb{C})}(f)$.

THEOREM 1.5 (Exponential sums around $Z$ ). If $f \in \mathcal{O}\left[x_{1}, \ldots, x_{n}\right]$ is a nonconstant polynomial and $Z$ is any closed subscheme of $\mathbb{A}_{\mathcal{O}}^{n}$, then there exist $c>0$ and $M>0$ such that

$$
\left|E_{f, L, \psi}^{Z}\right|<c m_{\psi}^{n-1} q_{L}^{-\sigma_{Z}(f) m_{\psi}}
$$

for all local fields $L$ over $\mathcal{O}$ whose residue field characteristic is at least $M$ and for all nontrivial additive characters $\psi$ on L satisfying $m_{\psi} \geqslant 2$.

The variant of Theorem 1.5 with $Z=\{0\}$ is the Denef-Sperber conjecture from [15], with the log canonical threshold in the exponent. Theorem 1.5 with $Z=\mathbb{A}_{\mathcal{O}}^{n}$ covers the variant [10, Conjecture 1.2 (1.2.1)] of Igusa's conjecture. At the end of Section 3, we will state and prove a version of Theorem 1.5 which is moreover uniform in the choice of $Z$, thus solving and generalizing the complete [10, Conjecture 1.2]. We moreover show the optimality of these estimates in the case of nonrational singularities by providing lower bounds in Section 3.4, where we also formulate the remaining part of Igusa's conjecture with precise and optimal estimates with the so-called motivic oscillation index of $f$ around $Z$ in the exponent. We also give an application of Theorem 1.1 about poles of maximal possible order of Igusa's local zeta functions in the twisted case; see Section 3.3.

1.3. Remarks on Igusa's conjecture. Igusa [26, pages 2 and 170] originally imposed two extra conditions in his conjecture: he focused on the case of homogeneous $f$ (mainly because in that case, 0 is the unique critical value), and he assumed $\sigma>1$ in (1.2), since he wanted to derive adèlic $L^{1}$-integrability of an adèlic variant of $E_{f, L, \psi}$ from his conjectural bounds on exponential sums. In Igusa's situation, lower values of $\sigma$ in (1.2) yield only adèlic $L^{q}$-integrability for higher $q$.

The exponent $\sigma_{Z}(f)$ in Theorem 1.5 is not always optimal. However, it is optimal in the case that the hypersurface given by $f-b$ for some $b \in \mathbb{C}$ has some 
nonrational singularities on each open $V$ containing $Z_{\mathbb{C}}$. We show this optimality in two steps: we introduce the notion of motivic oscillation index of $f$ around $Z$, denoted by $\operatorname{moi}_{Z}(f)$ (as a variant of a notion of [7], in particular, with a different sign), we show lower bounds of $\left|E_{f, L, \psi}^{Z}\right|$ with $\operatorname{moi}_{Z}(f)$ in the exponent instead of $\sigma_{Z}(f)$, and we compare the values of $\operatorname{moi}_{Z}(f)$ with $\sigma_{Z}(f)$ in the case of nonrational singularities. It may be interesting to study a relation between the motivic oscillation index and the notion of minimal exponents introduced in [39, page 52]. In the nonhomogeneous case, the case $m_{\psi}=1$ can be problematic if one uses the motivic oscillation index in the exponent, as witnessed by $f(x$, $y)=x^{2} y-x$; see [7, Example 7.2]. However, the case $m_{\psi}=1$ still makes sense by [7] for weighted homogeneous $f$ (and even possibly more generally, see the discussion following [4, Conjecture 1.2.2]). For general $f$, it is more sensible to restrict to $m_{\psi} \geqslant 2$, as observed in [10], where, moreover, the case $m_{\psi} \leqslant 4$ is proved.

Let us now discuss some previously proved cases of Igusa's conjecture on exponential sums and its variants. The case of the above theorems where $\sigma_{Z}(f) \leqslant 1 / 2$ has been recently obtained in [5]. Igusa treated (optimally) the case of homogeneous polynomials $f$ having an isolated singularity at 0 ; see [26, Section 5.3]. For polynomials $f$ that are nondegenerate with respect to their Newton polyhedron at the origin, the exponential sums $E_{f, L, \psi}$ and $E_{f, L, \psi}^{\{0\}}$ are well understood; see [15], [6], [8], and [4]. Moreover, in the nondegenerate case, it is expected that the bounds from $[4,6,8,15]$ are optimal; see the questions about optimality and a certain vertex condition from [14, Theorems 5.17-5.19] and [18]. For homogeneous polynomials in three variables and for weighted homogeneous polynomials in two variables, see [32, 33, 42]. It is most likely that Lichtin's method based on good wedge decomposition from $[32,33]$, in combination with Corollary 2.10 and with Cochrane's bounds from [11, Theorem 1], can also be used to yield Theorem 1.5, and a similar remark holds for the method of [5] based on arc spaces; both these approaches can probably avoid the use of multiplicative characters.

\section{Proof of Theorem 1.1}

2.1. Log resolutions. We first fix some terminology for log resolutions based on [17]. Let $K$ be a subfield of $\mathbb{C}$ and $X_{K}$ a smooth, geometrically connected variety over $K$. In the applications to Igusa's conjectures, we will take $X_{K}=\mathbb{A}_{K}^{n}$, but in this section, it is convenient to set up the notation in a more general setting. For a field extension $K^{\prime}$ of $K$, we put $X_{K^{\prime}}=X_{K} \times_{\operatorname{Spec}(K)} \operatorname{Spec}\left(K^{\prime}\right)$. Given a nonzero, noninvertible $f \in \mathcal{O}_{X_{K}}\left(X_{K}\right)$, we denote by $D_{K}$ the closed subscheme of $X_{K}$ defined by $f$ and put $D=D_{\mathbb{C}}$. Let $h: Y \rightarrow X_{K}$ be a log resolution of 
the pair $\left(X_{K}, D_{K}\right)$ (the existence of such $h$ follows from [17, page 142, Main Theorem II]).

More precisely, we have the following:

- $Y$ is a smooth closed subscheme of $\mathbb{P}_{X_{K}}^{k}$, for some $k \geqslant 0$.

- $h$ is a proper birational morphism which is an isomorphism over the complement of the support of $D_{K}$.

- The divisor $h^{*}\left(D_{K}\right)$ on $Y$ equals $\sum_{j \in T_{K}} N_{j} E_{j}$ for a finite set $T_{K}$ and some positive integers $N_{j}$, where each $E_{j}$ is a prime divisor.

- The divisor $h^{*}\left(D_{K}\right)$ has simple normal crossings, that is, if $I \subseteq T_{K}$ is such that $i \in I$ if and only if $a \in E_{i}$ and if we write in some neighborhood $V$ of $a$

$$
f \circ h_{\mid V}=u \prod_{i \in I} y_{i}^{N_{i}},
$$

with $y_{i} \in \mathcal{O}_{Y}(V)$ an equation of $E_{i}$ in $V$ and $u \in \mathcal{O}_{Y}(V)$ invertible, then the images of $\left(y_{i}\right)_{i \in I}$ in the stalk $\mathcal{O}_{Y, a}$ at $a$ are part of a regular system of parameters.

- The relative canonical divisor $K_{Y / X_{K}}$, which is locally defined by the determinant of the Jacobian matrix of $h$, is written as $\sum_{j \in T_{K}}\left(v_{j}-1\right) E_{j}$ for some positive integers $v_{j}$.

For any subset $I \subseteq T_{K}$, we put $E_{I}:=\bigcap_{i \in I} E_{i}$ if $I$ is nonempty and $E_{I}=Y$ if $I$ is the empty set. Further, we write

$$
E_{I}^{\circ}:=E_{I} \backslash \bigcup_{i \notin I} E_{i}
$$

By the functoriality of log resolutions for extensions of the base field, for any field $K^{\prime}$ containing $K, h$ induces a $\log$ resolution $h_{K^{\prime}}: Y_{K^{\prime}} \rightarrow X_{K^{\prime}}$ of the pair $\left(X_{K^{\prime}}, D_{K^{\prime}}\right)$. We note that each irreducible component $E_{i}$ for $i \in T_{K}$ splits into a disjoint union of finitely many irreducible components $E_{i j}$ over $K^{\prime}$ with $(i$, $j) \in T_{K^{\prime}}$ for a corresponding finite set $T_{K^{\prime}}$, and where we always have $N_{i}=N_{i j}$ and $v_{i}=v_{i j}$.

When $K^{\prime}=\mathbb{C}$, we write $J$ for $T_{K^{\prime}}$. We say that $I^{\prime} \subseteq J$ corresponds to $I \subseteq T_{K}$ if $I^{\prime}$ ranges over the irreducible components (over $\mathbb{C}$ ) of the $E_{i}$ for $i \in I$.

Definition 2.1 (Power condition for $(f, h, Z)$ ). Suppose now that $K=\mathbb{C}$ and let $f$ and $h: Y \rightarrow X$ be as above with $X=X_{\mathbb{C}}$. Let $Z$ be a closed subvariety of $X$ such that $f$ vanishes on $Z(\mathbb{C})$. Consider a nonempty open subset $W$ of an 
irreducible component of $E_{I}$ for some $I \subseteq J$, let $g$ be in $\mathcal{O}_{W}(W)$ and $d>1$ be an integer. We say that $(f, h, Z)$ satisfies the power condition, witnessed by $(I, W, g, d)$, if the following conditions hold:

$$
\begin{gathered}
h(W) \subseteq Z, \\
d \mid N_{i} \quad \text { for all } i \in I,
\end{gathered}
$$

and

$$
\left.u\right|_{W}=g^{d}
$$

where $u$ comes from writing $f \circ h=u \prod_{i \in I} y_{i}^{N_{i}}$ as in (2.1) on an open subset $V \subseteq Y$ with $W=E_{I} \cap V$. We simply say that the power condition holds for $(f$, $h, Z)$ if there exists $(I, W, g, d)$ witnessing the power condition for $(f, h, Z)$.

2.2. The proof of the log canonical threshold bound. Before giving the proof of Theorem 1.1, we make a few preliminary remarks using the notation in Section 1.1.

REMARK 2.2. In order to prove the inequality (1.5) in Theorem 1.1, it is enough to consider the case when $I$ has only one element. Indeed, given an arbitrary subset $I$ as in the theorem, let $Z$ be a connected component of $E_{I}$ that meets $X_{0}$ and let $h^{\prime}: Y^{\prime} \rightarrow Y$ be the blow-up of $Y$ along $Z$, with exceptional divisor $F$. Note that in this case, we have

$$
N=\operatorname{ord}_{F}(f)=\sum_{i=1}^{m} N_{i} \quad \text { and } \quad v=\operatorname{ord}_{F}\left(K_{Y^{\prime} / X}\right)+1=\sum_{i=1}^{m} v_{i} .
$$

Consider the chart $X_{0}^{\prime} \subseteq h^{\prime-1}\left(X_{0}\right)$ on $Y^{\prime}$ with coordinates $y_{1}, \ldots, y_{n}$ such that $x_{1}=y_{1}, x_{i}=y_{1} y_{i}$ for $2 \leqslant i \leqslant m$ and $x_{i}=y_{i}$ for $i>m$. In this case, we have

$$
\left.f \circ h \circ h^{\prime}\right|_{X_{0}^{\prime}}=y_{1}^{N} \cdot \prod_{i=2}^{m} y_{i}^{N_{i}} \cdot\left(u \circ h^{\prime}\right) .
$$

If $g^{\prime}=g \circ h^{\prime} \in \mathcal{O}\left(X_{0}^{\prime} \cap F\right)$, then $\left.u \circ h^{\prime}\right|_{X_{0}^{\prime} \cap F}=g^{\prime d}$ and clearly $d$ divides $N$. If we know (1.5) in the case of one divisor, we obtain

$$
\operatorname{lct}(f) \leqslant \frac{1}{d}+\sum_{i=1}^{m} v_{i}-\operatorname{lct}(f) \cdot \sum_{i=1}^{m} N_{i}=\frac{1}{d}+\sum_{i=1}^{m} N_{i}\left(\frac{v_{i}}{N_{i}}-\operatorname{lct}(f)\right) ;
$$

hence, (1.5) holds.

From now on, we will thus assume that $I$ contains only one element, corresponding to the divisor $E$ on $Y$, and denote by $N$ and $v$ the corresponding 
invariants. Note that in this case, the inequality in (1.5) is equivalent to

$$
\operatorname{lct}(f) \leqslant \frac{d v+1}{d(N+1)} .
$$

REMARK 2.3. It is interesting to note that in the case of one divisor $E$, the hypothesis on $f$ is independent of the $\log$ resolution $h$ and only depends on the valuation $v=\operatorname{ord}_{E}$ corresponding to $E$. Indeed, if $\mathcal{O}_{v}$ is the corresponding discrete valuation ring (DVR), with residue field $k_{v}$, and if we write $f=\pi^{N} u$, where $\pi$ is a uniformizer of $\mathcal{O}_{v}, N=v(f)$, and $u \in \mathcal{O}_{v}$ is invertible, then the condition on $f$ is that the class $\bar{u} \in k_{v}^{\times}$lies in $\left(k_{v}^{\times}\right)^{d}$ for some $d>1$ with $d \mid N$, where $\left(k_{v}^{\times}\right)^{d}$ is the set of $d$ th powers in $k_{v}^{\times}$.

REMARK 2.4. It is enough to prove (1.5) since it implies (1.6) for any $Z$ that satisfies the conditions in the theorem. Indeed, arguing as in Remark 2.2, we see that it is enough to consider the case when $I$ consists of only one element, corresponding to the divisor $E$, in which case the condition is that $h(E) \cap Z \neq \emptyset$. If $V$ is an open neighborhood of $Z$ such that $\operatorname{lct}_{Z}(f)=\operatorname{lct}\left(\left.f\right|_{V}\right)$, then $E \cap$ $h^{-1}(V) \neq \emptyset$, and we may apply the theorem for the restriction of $h$ over $V$ to obtain our assertion.

REMARK 2.5. We may and will assume that $E$ is an exceptional divisor. Indeed, otherwise we have $v=1$ and

$$
\operatorname{lct}(f) \leqslant \frac{1}{N} \leqslant \frac{d+1}{d(N+1)},
$$

where the second inequality follows from the fact that by assumption, we have $d \leqslant N$.

REMARK 2.6. The inequality (2.5) clearly holds if the right-hand side is $\geqslant 1$. We, thus, may and will assume that $(d v+1) /(d N+d)<1$; hence, $v<N+$ $(d-1) / d$. Since both $v$ and $N$ are integers, this implies $v \leqslant N$.

REMARK 2.7. Furthermore, we may also assume that there is a rational number $c$, with $0<c<\operatorname{lct}(f)$, such that

$$
v-c \cdot N<1 .
$$

The existence of such $c$ is clear if $(v-1) / N<\operatorname{lct}(f)$. On the other hand, if $\operatorname{lct}(f) \leqslant(v-1) / N$, then we are done since it is easy to check that we have

$$
\frac{v-1}{N}<\frac{d v+1}{d N+d}
$$

when $v \leqslant N$. 
The existence of such $c$ as above is useful since it implies that there is a projective, birational morphism $\pi: W \rightarrow X$, with $W$ normal, such that $E$ appears as a prime $\mathbb{Q}$-Cartier divisor $E_{W}$ on $W$ and such that $E_{W}$ is the unique exceptional divisor of $\pi$. This is a well-known consequence of the Minimal Model Program: note that the pair $(X, c D)$ is klt and we can apply [2, Corollary 1.4.3] or [3, Propositions 3.2 and 4.1]. Furthermore, since $X$ is smooth, hence $\mathbb{Q}$-factorial, the exceptional locus of $\pi$ has pure codimension 1 ; hence, it is equal to $E_{W}$. Note that while $W$ is not a $\log$ resolution of $(X, D)$, the hypothesis in the theorem is birational with respect to the divisor; hence, it also holds for $E_{W}$ (see Remark 2.3).

The assertion in the following lemma is well known, but we include a proof for the sake of completeness.

Lemma 2.8. If $X$ is a normal, $\mathbb{Q}$-Gorenstein variety and $Z \subseteq X$ is a codimension 2 irreducible closed subset, such that $X$ is not smooth at the generic point of $Z$, then there is a projective, birational morphism $\pi: \widetilde{X} \rightarrow X$, with $\widetilde{X}$ smooth, and a prime divisor $F$ on $\widetilde{X}$ such that $\pi(F)=Z$ and the coefficient of $F$ in $K_{\tilde{X} / X}$ is $\leqslant 0$.

Proof. Let $\pi: \widetilde{X} \rightarrow X$ be a $\log$ resolution, with exceptional divisor $F_{1}+\cdots+F_{r}$. It follows from [30, Corollary 2.32] that if $Y$ is the union of those $\pi\left(F_{i}\right)$ such that $F_{i}$ has coefficient $\leqslant 0$ in $K_{\widetilde{X} / X}$ and $U=X \backslash Y$, then $U$ has terminal singularities. In particular, $U$ is smooth in codimension 2 (see [30, Corollary 5.18]); hence, our assumption implies that $Z \subseteq Y$. Therefore, $Z$ is an irreducible component of $Y$; hence, it is equal to $\pi\left(F_{i}\right)$ for some divisor $F_{i}$ whose coefficient in $K_{\widetilde{X} / X}$ is $\leqslant 0$.

Finally, we will need the following bound for the intersection multiplicity of two curves.

LemMA 2.9. Let $\left(R, \mathfrak{m}_{R}\right)$ be a local, excellent domain, with $\operatorname{dim}(R)=2$. Suppose that $g \in \mathfrak{m}_{R}$ is a nonzero element that generates a prime ideal and $h \in \mathfrak{m}_{R}$ is such that its image $\bar{h}$ in $A=R /(g)$ is nonzero and can be written as $u^{d}$, for some $u$ in the fraction field of $A$. In this case, we have

$$
\ell_{R}(R /(g, h)) \geqslant d
$$

Proof. Let $B$ be the integral closure of $A$ in its fraction field. Since $R$ is excellent, $B$ is a finitely generated $A$-module. For a finitely generated $A$-module $M$ and an ideal $\mathfrak{q}$ in $A$, with radical equal to the maximal ideal, we write $e_{A}(\mathfrak{q}, M)$ for the 
Samuel multiplicity of $M$ with respect to $\mathfrak{q}$. Since $\operatorname{rank}_{A}(B)=1$, it follows from [34, Theorem 14.8] that

$$
e_{A}((\bar{h}), B)=e_{A}((\bar{h}), A)
$$

Note also that we have

$$
e_{A}((\bar{h}), A)=\ell_{A}(A /(\bar{h}))=\ell_{R}(R /(g, h)),
$$

where the first equality follows from the fact that $\bar{h}$ is a nonzero divisor in the one-dimensional local ring $A$ (see [34, Theorem 14.11]). Using the fact that $\bar{h}$ and $u$ are nonzero divisors in $B$, we also have

$$
e_{A}((\bar{h}), B)=\ell_{A}(B / \bar{h} B)=d \cdot \ell_{A}(B / u B) \geqslant d .
$$

This completes the proof of the lemma.

We can now give the proof of the bound for the log canonical threshold.

Proof of Theorem 1.1. We may and will assume that we are in the situation described in Remark 2.7, with a morphism $\pi: W \rightarrow X$ whose exceptional locus is equal to $E_{W}$, the prime divisor on $W$ corresponding to $E$. By hypothesis, $h$ is an isomorphism over $X \backslash D$; hence, $\pi\left(E_{W}\right) \subseteq D$. For every $y \in \pi\left(E_{W}\right)$, the fiber $\pi^{-1}(y)$ is contained in the exceptional locus. Since $\pi$ is proper, it follows that $\pi^{-1}(y) \cap \widetilde{D} \neq \emptyset$, where $\widetilde{D}$ is the strict transform of $D$ on $W$. In particular, $E_{W} \cap \widetilde{D}$ is nonempty. Note that we have $\pi^{*}(D)=\widetilde{D}+N E_{W}$ and $E_{W}$ is $\mathbb{Q}$ Cartier; hence, the divisor $\widetilde{D}$ is $\mathbb{Q}$-Cartier. Similarly, since $K_{W / X}=(v-1) E_{W}$, it follows that $K_{W / X}$ is $\mathbb{Q}$-Cartier.

Let $c=\operatorname{lct}(f)$ so that $(X, c D)$ is $\log$ canonical. This implies that also the pair

$$
\left(W, c \cdot \pi^{*}(D)-K_{W / X}\right)=\left(W, c_{1} \widetilde{D}+c_{2} E_{W}\right)
$$

is $\log$ canonical, where $c_{1}=c$ and $c_{2}=c N-v+1$. Arguing by contradiction, we may assume that $c>(d v+1) /(d N+d)$. In this case, we have

$c_{1}+c_{2}=c+(c N-v+1)=c(N+1)-v+1>\frac{d v+1}{d}-v+1=1+\frac{1}{d}$.

In particular, this gives $c_{2}>1 / d>0$.

We now consider a suitable cyclic cover. Let $s$ be a positive integer such that $s E_{W}$ is Cartier and choose an open subset $V$ of $W$ meeting $\widetilde{D} \cap E_{W}$ such that we have an isomorphism $\mathcal{O}_{V}\left(s E_{V}\right) \simeq \mathcal{O}_{V}$, where $E_{V}=\left.E_{W}\right|_{V}$. After possibly replacing $s$ by a divisor and $V$ by a smaller open subset, we may assume that 
$s^{\prime} E_{V}$ is not Cartier for any divisor $s^{\prime}$ of $s$ different from $s$. Consider the $\mathcal{O}_{V^{-}}$ algebra

$$
\mathcal{A}=\mathcal{O}_{V} \oplus \mathcal{O}_{V}\left(E_{V}\right) \oplus \cdots \oplus \mathcal{O}_{V}\left((s-1) E_{V}\right),
$$

where multiplication is defined using the fact that for $0 \leqslant i, j \leqslant s-1$ with $i+j \geqslant s$, we have

$$
\mathcal{O}_{V}\left(i E_{V}\right) \otimes \mathcal{O}_{V}\left(j E_{V}\right) \rightarrow \mathcal{O}_{V}\left((i+j) E_{V}\right) \simeq \mathcal{O}\left((i+j-s) E_{V}\right) .
$$

Note that we have a finite surjective morphism $\varphi: U=\operatorname{Spec}(\mathcal{A}) \rightarrow V$. It is well known and straightforward to check that $U$ is normal and $\varphi$ is étale in codimension 1; in particular, we have $K_{U / V}=0$. Moreover, the section 1 of $\mathcal{O}_{V}\left(E_{V}\right)$ defines an effective Cartier divisor $E_{U}$ on $U$ such that $\varphi^{*}\left(E_{V}\right)=E_{U}$. We also put $D_{U}=\varphi^{*}(\widetilde{D})$. Note that since $\widetilde{D}+N E_{W}$ is Cartier and $E_{U}$ is Cartier, it follows that $D_{U}$ is Cartier as well. Furthermore, since $\widetilde{D} \cap E_{V} \neq \emptyset$, we conclude that $D_{U} \cap E_{U} \neq \emptyset$. Let $Z$ be an irreducible component of $D_{U} \cap E_{U}$ so that $Z$ has codimension 2 in $U$.

We first show that $U$ is smooth at the generic point of $Z$. Indeed, since $Z$ has codimension 2 in $X$, if $U$ is not smooth at the generic point of $Z$, it follows from Lemma 2.8 that there is a prime divisor $F$ on some smooth variety $\widetilde{U}$, with a birational morphism $\widetilde{U} \rightarrow U$, such that $F$ dominates $Z$ and $\operatorname{ord}_{F}\left(K_{\widetilde{U} / U}\right) \leqslant 0$. Since $K_{U / V}=0$ and the pair $\left(W, c_{1} \widetilde{D}+c_{2} E_{W}\right)$ is log canonical, it follows that the pair $\left(U, c_{1} D_{U}+c_{2} E_{U}\right)$ is $\log$ canonical, and, thus,

$$
1 \geqslant 1+\operatorname{ord}_{F}\left(K_{\widetilde{U} / U}\right) \geqslant c_{1} \cdot \operatorname{ord}_{F}\left(D_{U}\right)+c_{2} \cdot \operatorname{ord}_{F}\left(E_{U}\right) \geqslant c_{1}+c_{2}>1+\frac{1}{d},
$$

a contradiction. Therefore, $U$ is smooth at the generic point of $Z$.

Note that since $\varphi$ is étale in codimension 1, the divisor $E_{U}$ is reduced. Let $E_{U}^{1}$, $\ldots, E_{U}^{r}$ be the prime divisors containing $Z$ that appear in $E_{U}$. If $R=\mathcal{O}_{U, Z}$, then the image of $f$ in $R$ factors as $h \cdot \prod_{i=1}^{r} g_{i}^{N}$, where $h \in R$ is a local equation of $D_{U}$ and $g_{1}, \ldots, g_{r} \in R$ are local equations of $E_{U}^{1}, \ldots, E_{U}^{r}$. By considering the local homomorphism $\mathcal{O}_{V, E_{V}} \hookrightarrow \mathcal{O}_{U, E_{U}^{1}}$, we deduce from the hypothesis in the theorem that the class of $h \cdot \prod_{i=2}^{r} g_{i}^{N}$ in $k\left(E_{U}^{1}\right)$ is the $d$ th power of some element of $k\left(E_{U}^{1}\right)$; therefore, the same holds for the class of $h$ in $k\left(E_{U}^{1}\right)$. We may thus apply Lemma 2.9 to conclude that

$$
\ell_{R}\left(R /\left(g_{1}, h\right)\right) \geqslant d .
$$

On the other hand, since $U$ is smooth at the generic point of $Z$, we have the divisorial valuation $\operatorname{ord}_{Z}$ of the function field of $U$ (this corresponds to the exceptional divisor on the blow-up along $Z$ of a smooth open subset of $U$ 
meeting Z). Since the pair $\left(U, c_{1} D_{U}+c_{2} E_{U}\right)$ is $\log$ canonical, we have

$$
2 \geqslant c_{1} \cdot \operatorname{ord}_{Z}\left(D_{U}\right)+c_{2} \cdot \operatorname{ord}_{Z}\left(E_{U}\right) .
$$

Since $c_{1}+c_{2}>1$, we conclude that either $\operatorname{ord}_{Z}\left(E_{U}\right)=1$ or $\operatorname{ord}_{Z}\left(D_{U}\right)=1$. We treat these two cases separately.

Case 1. Suppose that $\operatorname{ord}_{Z}\left(E_{U}\right)=1$, that is, $E_{U}$ is smooth at the generic point of $Z$. In particular, we have $r=1$. In this case, there is a regular system of parameters of $R$ given by $g_{1}$ and some $x \in R$. Let $v$ be the monomial valuation (with respect to this coordinate system) of the fraction field of $R$ such that $v\left(g_{1}\right)=d$ and $v(x)=1$. Condition (2.7) implies that $h \in\left(g_{1}, x^{d}\right)$; hence, $v(h) \geqslant d$. It is a standard fact that the $\log$ discrepancy of $v$ is $d+1$; hence, the fact that $\left(U, c_{1} D_{U}+c_{2} E_{U}\right)$ is log canonical implies

$$
d+1 \geqslant c \cdot v(h)+(c N-v+1) \cdot v\left(g_{1}\right) \geqslant d(c+c N-v+1) .
$$

A straightforward computation then gives

$$
c \leqslant \frac{d v+1}{d N+d}
$$

completing the proof of this case.

Case 2. If $\operatorname{ord}_{Z}\left(D_{U}\right)=1$, we proceed similarly. Consider a regular system of parameters of $R$ given by $h$ and $y$ and consider the monomial valuation $w$ (in this system of coordinates) of the fraction field of $R$ such that $w(h)=d$ and $w(y)=1$. It follows from (2.7) that $g_{1} \in\left(h, y^{d}\right)$; hence, $w\left(g_{1}\right) \geqslant d$. Since the $\log$ discrepancy of $w$ is $d+1$, using the fact that the pair $\left(U, c_{1} D_{U}+c_{2} E_{U}\right)$ is $\log$ canonical, we obtain

$$
d+1 \geqslant c \cdot w(h)+(c N-v+1) \cdot w\left(g_{1}\right) \geqslant d c+d(c N-v+1),
$$

which again implies $c \leqslant(d v+1) /(d N+d)$, completing the proof of the theorem.

2.3. A special case. For our applications, we will use the following corollary of Theorem 1.1, with notation as above and with $X=\mathbb{A}_{\mathbb{C}}^{n}$.

COROLlaRY 2.10. Let $f$ be a nonconstant polynomial in $\mathbb{C}[x], h: Y \rightarrow \mathbb{A}_{\mathbb{C}}^{n}$ a log resolution of the pair $\left(\mathbb{A}_{\mathbb{C}}^{n}, D\right)$ with $D$ given by $f$ and let $Z$ be a closed subvariety of $\mathbb{A}_{\mathbb{C}}^{n}$ such that $f$ vanishes on $Z(\mathbb{C})$. If the power condition holds for $(f, h, Z)$, witnessed by some $(I, W, g, d)$, then the following inequality holds:

$$
\operatorname{lct}_{Z}(f) \leqslant \frac{1}{d}+\sum_{i \in I}\left(v_{i}-N_{i} \cdot \operatorname{lct}_{Z}(f)\right) .
$$


Note that the weaker inequality

$$
\operatorname{lct}_{Z}(f) \leqslant \frac{1}{2}+\sum_{i \in I}\left(v_{i}-N_{i} \cdot \operatorname{lct}_{Z}(f)\right),
$$

with $1 / 2$ instead of the term $1 / d$ in (2.8), would already suffice to prove our results on exponential sums but not for our application on poles with largest possible multiplicity in Section 3.3.

\section{Igusa's local zeta function and exponential sums}

3.1. Preliminaries on exponential sums. Let $f, Z$, and $\mathcal{O}$ be as in Theorem 1.5. Consider a local field $L$ over $\mathcal{O}$ and let $\chi: \mathcal{O}_{L}^{\times} \rightarrow \mathbb{C}^{\times}$be a multiplicative character, that is, a continuous group homomorphism on the group of units, $\mathcal{O}_{L}^{\times}$, of $\mathcal{O}_{L}$. Note that any such $\chi$ has a finite image. The order of $\chi$ is the number of elements in its image. The conductor $c(\chi)$ of $\chi$ is the smallest $c \geqslant 1$ for which $\chi$ is trivial on $1+\mathcal{M}_{L}^{c}$, with $\mathcal{M}_{L}$ the maximal ideal of $\mathcal{O}_{L}$. We put $\chi(0)=0$. Let $s$ be a complex number with real part at least 0 . With a fixed uniformizer $\varpi_{L}$ of $\mathcal{O}_{L}$, we consider the map ac: $L \rightarrow \mathcal{O}_{L}$ that sends a nonzero $x$ to $x \varpi_{L}^{- \text {ord } x}$ and 0 to 0 . Further, write $\overline{\mathrm{ac}}(x)$ in $k_{L}$ for the reduction of ac $(x)$ modulo $\mathcal{M}_{L}$. We now associate to this data Igusa's local zeta function

$$
\mathcal{Z}_{f, L, \chi, s}^{Z}:=\int_{\left\{x \in \mathcal{O}_{L}^{n} \mid \bar{x} \in Z\left(k_{L}\right)\right\}} \chi(\operatorname{ac}(f(x)))|f(x)|^{s}|d x| .
$$

Igusa showed in [21] that $\mathcal{Z}_{f, L, \chi, s}^{Z}$ is a rational function in $t=q_{L}^{-s}$ when $L$ has characteristic zero (and, when $L$ has positive, large enough characteristic, given $f$ ), thus starting the study of a now vast subject.

We begin by recalling a result relating exponential sums to Igusa's local zeta functions (see [13, Proposition 1.4.4]).

Proposition 3.1. Let $f, \mathcal{O}$, and $Z$ be as in Theorem 1.5, L be a local field over $\mathcal{O}$, and $\psi$ be a nontrivial additive character on $L$. If we put $m=m_{\psi}, q=q_{L}$, and $t=q^{-s}$, then $E_{f, L, \psi}^{Z}$ is equal to

$$
\mathcal{Z}_{f, L, 1,0}^{Z}+\operatorname{Coeff}_{t^{m-1}}\left(\frac{(t-q) \mathcal{Z}_{f, L, 1, s}^{Z}}{(q-1)(1-t)}\right)+\sum_{\chi \neq 1} g_{\chi^{-1}, \psi} \operatorname{Coeff}_{t^{m-c(x)}}\left(\mathcal{Z}_{f, L, \chi, s}^{Z}\right),
$$

where 1 stands for the trivial character on $\mathcal{O}_{L}^{\times}$, the summation index $\chi$ runs over all nontrivial multiplicative characters on $\mathcal{O}_{L}^{\times}, g_{\chi, \psi}$ is a complex number 
depending only on $\chi$ and $\psi$, and $\operatorname{Coeff}_{t} S(t)$ for any $\ell \geqslant 0$ and any power series $S$ in $t$ stands for the coefficient of $t^{\ell}$. Moreover, if $c(\chi)=1$, then

$$
\left|g_{\chi, \psi}\right|=\frac{q^{1 / 2}}{q-1} .
$$

For an explicit description of the $g_{\chi, \psi}$ in (3.2), see [13, Proposition 1.4.4], whose proof applies to local fields of any characteristic.

We recall a variant of the Lang-Weil estimates and a corollary.

Proposition 3.2 (Lang-Weil estimates). Let $k=\mathbb{F}_{q}$ be a finite field and $X \subseteq$ $\mathbb{P}_{k}^{n}$ be a closed subvariety of dimension $r$. If $X$ is geometrically irreducible, then there is a positive constant $c_{X}$ such that for every $\ell \geqslant 1$, we have

$$
\left|\# X\left(\mathbb{F}_{q^{\ell}}\right)-q^{\ell r}\right| \leqslant c_{X} q^{\ell(r-1 / 2)} .
$$

Moreover, $c_{X}$ can be taken independently from $X$ and from $q$ as long as $n, r$, and the number and degrees of the equations defining $X$ remain bounded.

Proof. The existence of $c_{X}$ comes from the usual Lang-Weil estimates. The independence of $c_{X}$ from $X$ and from $q$ (as long as the complexity of $X$ stays bounded) follows from [28, Theorem 12], which gives furthermore explicit upper bounds for $c_{X}$ in terms of the complexity of $X$ (see also [38, Theorem 3.1]).

COROLlary 3.3. Let $\mathcal{O}$ be a ring of integers and let $d>1$ be an integer. Let $X \subseteq \mathbb{A}_{\mathcal{O}}^{n}$ be a closed subscheme such that $X_{\mathbb{C}}$ is an irreducible closed subvariety of $\mathbb{A}_{\mathbb{C}}^{n}$ of dimension $r$, and let $F: X \rightarrow \mathbb{A}_{\mathcal{O}}^{1}$ be a regular morphism such that $F$ is nonvanishing on $X(\mathbb{C})$. Suppose that there does not exist $e>1$ dividing $d$ and $a$ regular morphism $g: V \subset X_{\mathbb{C}} \rightarrow \mathbb{A}_{\mathbb{C}}^{1}$ on a nonempty open $V$ of $X_{\mathbb{C}}$ such that $g^{e}$ equals $\left.F\right|_{V}$. Then there exist constants $c$ and $M$ such that for all finite fields $\mathbb{F}_{q}$ of characteristic at least $M$ with $\mathbb{F}_{q}$ an algebra over $\mathcal{O}$, and for any character $\chi$ of $\mathbb{F}_{q}^{\times}$of orderd, we have

$$
\left|\sum_{x \in X\left(\mathbb{F}_{q}\right)} \chi(F(x))\right| \leqslant c q^{r-1 / 2} .
$$

Moreover, c can be taken independently from $X$ and $F$ as long as $n, r, d$, and the number and degrees of the equations defining $X$ and $F$ remain bounded.

Proof. Let $U$ be the Kummer cover of $X$ given by $F(x)=y^{d}$ for $x \in X$. By our assumptions, we have that $U_{\mathbb{C}}$ is irreducible, and, thus, there exist $M$ and $c$ such 
that for each finite field $\mathbb{F}_{q}$, which is an algebra over $\mathcal{O}$ and whose characteristic is at least $M$, we have that $U_{\mathbb{F}_{q}}$ is geometrically irreducible (for example, by model theoretic compactness) and, by Proposition 3.2, that

$$
\left|\# U\left(\mathbb{F}_{q}\right)-q^{\operatorname{dim} X}\right| \leqslant c q^{\operatorname{dim} X-1 / 2} .
$$

Write $\mathbb{F}_{q}^{\times, d}$ for the set of $d$ th powers in $\mathbb{F}_{q}^{\times}$and $d_{q}$ for the index of $\mathbb{F}_{q}^{\times, d}$ in $\mathbb{F}_{q}^{\times}$. Clearly, we also have

$$
\frac{\# U\left(\mathbb{F}_{q}\right)}{d_{q}}=\#\left\{x \in X\left(\mathbb{F}_{q}\right) \mid F(x) \text { is a } d \text { th power in } \mathbb{F}_{q}^{\times}\right\} .
$$

Similarly, for each such $q$ and for each $\lambda \in \mathbb{F}_{q}^{\times}$, we consider $U_{\lambda}$ given by $F(x)=$ $\lambda y^{d}$. By the uniformity of the constant in Proposition 3.2, we can choose $M$ and $c$ as above and such that, in addition, if the characteristic of $\mathbb{F}_{q}$ is at least $M$ (and if $\mathbb{F}_{q}$ is an algebra over $\mathcal{O}$ ), then for each $\lambda \in \mathbb{F}_{q}^{\times}$we have that $U_{\lambda}$ is geometrically irreducible (again, by model theoretic compactness) and

$$
\mid \#\left\{x \in X\left(\mathbb{F}_{q}\right) \mid \frac{F(x)}{\lambda} \text { is a } d \text { th power in } \mathbb{F}_{q}^{\times}\right\}-\frac{q^{\operatorname{dim} X}}{d_{q}} \mid \leqslant \frac{c}{d_{q}} q^{\operatorname{dim} X-1 / 2} .
$$

By orthogonality of characters, for any character $\chi$ of $\mathbb{F}_{q}^{\times}$of order $d$, we have

$$
\sum_{\bar{\lambda} \in \mathbb{F}_{q}^{\times} / \mathbb{F}_{q}^{\times, d}} \chi(\bar{\lambda})=0
$$

from which the corollary follows. Indeed, the required uniformity of $c$ comes from the uniformity in Proposition 3.2 and the fact that the complexity of the covers $U_{\lambda}$ is clearly bounded when $n, r, d$, and the number and degrees of the equations defining $X$ and $F$ are bounded.

We next give a combination of Denef's formula for Igusa's local zeta function, the Lang-Weil estimates, and Corollary 3.3. Possibly, one may use the more advanced estimates of [38, Theorem 1.1] on finite field exponential sums with multiplicative characters instead of Corollary 3.3. Let $K$ be the field of fractions of $\mathcal{O}$. With the notation in Section 2.1, with $X=\mathbb{A}_{K}^{n}$ and $f \in K[x]=K\left[x_{1}\right.$, $\left.\ldots, x_{n}\right]$ nonconstant, we consider $D_{K}$ and fix a log resolution $h: Y \rightarrow \mathbb{A}_{K}^{n}$ of the pair $\left(\mathbb{A}_{K}^{n}, D_{K}\right)$.

Proposition 3.4. Let $f, Z, \mathcal{O}$, and $h$ be as above. Assume, moreover, that $f$ vanishes on $Z(\mathbb{C})$. Then there exist constants $C$ and $M$ so that the following 
formula holds for every local field $L$ over $\mathcal{O}$ with residue field characteristic at least $M$ and every multiplicative character $\chi$ on $\mathcal{O}_{L}^{\times}$:

$$
\mathcal{Z}_{f, L, \chi, s}^{Z}=\sum_{I \subseteq T_{K}} c_{I, Z, L, \chi} \cdot \prod_{i \in I} \frac{q_{L}^{-N_{i} s-v_{i}}}{1-q_{L}^{-N_{i} s-v_{i}}},
$$

where the complex numbers $c_{I, Z, L, \chi}$ are independent of $s$ and satisfy

$$
\left|c_{I, Z, L, \chi}\right| \leqslant C \text {. }
$$

Moreover, for such L, we further have

$$
c_{I, Z, L, \chi}=0
$$

if $c(\chi)>1$ or if the order of $\chi$ does not divide $N_{i}$ for some $i \in I$. Furthermore, if $\chi$ is nontrivial and for $I^{\prime} \subseteq J$ corresponding to $I$, there exist no $W, g, d$ with $\left(f, h, \mathbb{A}^{n}\right)$ satisfying the power condition witnessed by $\left(I^{\prime}, W, g, d\right)$, then

$$
\left|c_{I, Z, L, \chi}\right| \leqslant C q_{L}^{-1 / 2} \text {. }
$$

Finally, given $f$ and $h$, the constants $C$ and $M$ can be taken independently from $Z$ and $\mathcal{O}$, as long as the number of equations defining $Z$ and their degrees remain bounded.

Proof. By [12, Theorem 2.1] (or, equivalently, [13, Theorem 3.3]), if $c(\chi)>1$, and since $f$ vanishes on $Z(\mathbb{C})$, we have $c_{I, Z, L, \chi}=0$ for all $I$ and all $Z$ (as soon as the residue field characteristic is large enough) and we are done for such $\chi$. Let us thus take $\chi$ with $c(\chi)=1$. The existence of the complex numbers $c_{I, Z, L, \chi}$ such that (3.5) holds as well as their independence of $s$ follow from Denef's formula [12, Theorem 2.2] (or, equivalently, [13, Theorem 3.4]), where also explicit descriptions of the $c_{I, Z, L, \chi}$ are given as finite exponential sums (as soon as the residue field characteristic is large enough). Precisely, the explicit description of the $c_{I, Z, L, \chi}$ given in [12, Theorem 2.1] (or [13, Theorem 3.3]) is as follows:

$$
c_{I, Z, L, \chi}=\frac{\left(q_{L}-1\right)^{\# I}}{q_{L}^{n}} \sum_{a \in E_{I}^{\circ}\left(k_{L}\right), h(a) \in Z\left(k_{L}\right)} \chi(u(a))
$$

if the order of $\chi$ divides $N_{i}$ for each $i \in I$ and if the characteristic of $k_{L}$ is sufficiently large (depending only on $f$ and $h$ ), where we take natural reductions modulo the maximal ideal $\mathcal{M}_{L}$ of $\mathcal{O}_{L}$ when we write $u(a), h(a)$, and $E_{I}^{\circ}\left(k_{L}\right)$. The bound (3.6) now follows from the Lang-Weil estimates for bounding the number of elements in $E_{I}^{\circ}\left(k_{L}\right)$. Further, if $I$ is such that the order of $\chi$ 
does not divide $N_{i}$ for some $i \in I$, then $c_{I, Z, L, \chi}=0$ by [12, Theorem 2.2] (or [13, Theorem 3.4]), still assuming that the residue field characteristic is large enough. This proves (3.7).

We still need to show (3.8). Suppose, thus, that $\chi$ is nontrivial and, given $I$, that there does not exist $W, g, d$ such that $\left(f, h, \mathbb{A}^{n}\right)$ satisfies the power condition witnessed by $\left(I^{\prime}, W, g, d\right)$, with $I^{\prime}$ corresponding to $I$. For those $L$ such that the reduction of $E_{I}$ modulo $\mathcal{M}_{L}$ has no irreducible component (defined over $k_{L}$ ) which is moreover geometrically irreducible, we reason as follows. By the smoothness of $E_{I}$, we have that the reduction of $E_{I}$ modulo $\mathcal{M}_{L}$ is also smooth as soon as $p_{L}$ is large. Hence, if no irreducible component of the reduction of $E_{I}$ modulo $\mathcal{M}_{L}$ is moreover geometrically irreducible, then there are no $k_{L}$-rational points on the reduction of $E_{I}$ by its smoothness, and (3.8) is clear. Suppose now that there is an irreducible component of $E_{I, k_{L}}$ (the reduction of $E_{I}$ modulo $\mathcal{M}_{L}$ ) which is geometrically irreducible. By working separately for each component of $E_{I, k_{L}}$ and using the Lang-Weil estimates in order to see that we can ignore algebraic subsets of codimension at least 1 , we can choose an affine open $V$ of $E_{I}^{\circ}$ and restrict the summation index in (3.9) by imposing both $a \in V\left(k_{L}\right)$ and $h(a) \in Z\left(k_{L}\right)$. Furthermore, since the power condition for $\left(f, h, \mathbb{A}^{n}\right)$ does not hold for any witnesses of the form $\left(I^{\prime}, W, g, d\right)$, with $I^{\prime}$ corresponding to $I$ and any integer $d>1$, we may apply Corollary 3.3 to the sum in (3.9) restricted to $a \in V\left(k_{L}\right)$ to find the bound from (3.8) in the case that $V_{k_{L}} \cap h^{-1}\left(Z_{k_{L}}\right)$ has dimension equal to $\operatorname{dim} E_{I}$ and Proposition 3.2 in the case that its dimension is less than $\operatorname{dim} E_{I}$, with $V_{k_{L}}$ and $Z_{k_{L}}$ denoting the reductions. The proposition is proved: note that the uniformity of $C$ and $M$ for varying $Z$ and $\mathcal{O}$ follows from the uniformity of Proposition 3.2 and Corollary 3.3.

3.2. Proofs of the estimates for exponential sums. Our strategy for proving Theorem 1.5 is to first reduce to the case when $f$ vanishes on $Z(\mathbb{C})$. Next, we relate the exponential sums to Igusa's local zeta functions using Proposition 3.1, and finally, we estimate the different parts in (3.2) of Proposition 3.1 using Proposition 3.4, Corollary 2.10, and the following two propositions. Let $f \in \mathcal{O}[x]$ and $Z$ be as in Theorem 1.5 and let $K$ be the number field which is the field of fractions of $\mathcal{O}$. We fix a $\log$ resolution $h: Y \rightarrow \mathbb{A}_{K}^{n}$ of $D_{K}$. In order to estimate the first term in (3.2), we use the following result from [5].

Proposition 3.5 [5, Lemma 4.1]. Given $f$ and $h$ as above, there exist positive constants $C$ and $M$ such that, for any integer $m \geqslant 2$, any ring of integers $\mathcal{O}_{1}$ containing $\mathcal{O}$, any closed subscheme $Z$ of $\mathbb{A}_{\mathcal{O}_{1}}^{n}$ such that $f$ vanishes on $Z(\mathbb{C})$, 
and any local field $L$ over $\mathcal{O}_{1}$ with $p_{L}$ larger than $M$, we have

$$
\left|\mathcal{Z}_{f, L, 1,0}^{Z}+\operatorname{Coeff}_{t^{m-1}} \frac{(t-q) \mathcal{Z}_{f, L, 1, s}^{Z}}{(q-1)(1-t)}\right| \leqslant C m^{n-1} q^{-m \cdot \operatorname{lct}_{Z}(f)},
$$

where $q=q_{L}$ and $t=q^{-s}$.

Proof. Lemma 4.1 of [5] states and proves this for $Z=\{0\}$, but in its proof, one can replace this $\{0\}$ by any choice of $Z$ such that $f$ vanishes on $Z(\mathbb{C})$ and $\operatorname{lct}_{0}(f)$ by $\operatorname{lct}_{Z}(f)$. Indeed, the estimates (4.7) and (4.9) of [5] are valid for any $Z$ such that $f$ vanishes on $Z(\mathbb{C})$ instead of $\{0\}$ and with $\operatorname{lct}_{Z}(f)$ instead of $\operatorname{lct}_{0}(f)$.

We now estimate the remaining parts in (3.2) of Proposition 3.1.

Proposition 3.6. Let $f, \mathcal{O}$, and $Z$ be as in Theorem 1.5. If $I \subseteq T_{K}$ is such that $h\left(E_{I}(\mathbb{C})\right) \cap Z(\mathbb{C})$ is nonempty, then for every $q>1$ and every $m \geqslant 2$, we have

$$
\left|\operatorname{Coeff}_{t^{m-1}}\left(\prod_{i \in I} \frac{t^{N_{i}} q^{-v_{i}}}{1-t^{N_{i}} q^{-v_{i}}}\right)\right| \leqslant q^{-(m-1) \operatorname{lct}(f)+\sigma_{I}} m^{\#(I)-1},
$$

where

$$
\sigma_{I}=-\sum_{i \in I}\left(v_{i}-N_{i} \operatorname{lct}_{Z}(f)\right)
$$

Proof. Since $h\left(E_{I}(\mathbb{C})\right) \cap Z(\mathbb{C})$ is nonempty, it follows that $v_{i} / N_{i} \geqslant \operatorname{lct}_{Z}(f)$ for all $i \in I$. We have

$$
\operatorname{Coeff}_{t^{m-1}} \prod_{i \in I} \frac{t^{N_{i}} q^{-v_{i}}}{1-t^{N_{i}} q^{-v_{i}}}=\sum_{\left(a_{i}\right)_{i \in I} \in A_{I, m}} q^{-\sum_{i \in I} v_{i}\left(a_{i}+1\right)},
$$

where

$$
A_{I, m}=\left\{\left(a_{i}\right)_{i \in I} \in \mathbb{N}^{\# I} \mid \sum_{i \in I} N_{i}\left(a_{i}+1\right)=m-1\right\} .
$$

For each $\left(a_{i}\right)_{i \in I} \in A_{I, m}$, we have

$$
\begin{aligned}
-\sum_{i \in I} v_{i}\left(a_{i}+1\right) & =-(m-1) \operatorname{lct}_{Z}(f)-\sum_{i \in I}\left(a_{i}+1\right)\left(v_{i}-N_{i} \operatorname{lct}_{Z}(f)\right) \\
& \leqslant-(m-1) \operatorname{lct}_{Z}(f)-\sum_{i \in I}\left(v_{i}-N_{i} \operatorname{lct}_{Z}(f)\right)
\end{aligned}
$$

where the inequality follows from the fact that $v_{i} / N_{i} \geqslant \operatorname{lct}_{Z}(f)$. Since \# $\left(A_{I, m}\right) \leqslant$ $m^{\#(I)-1}$, we obtain the assertion in the proposition. 
We can now prove our main results on exponential sums.

Proof of Theorem 1.5. Let $f, \mathcal{O}$, and $Z$ be as in Theorem 1.5. Let $V_{f}=\left\{z_{1}, \ldots\right.$, $\left.z_{e}\right\}$ be the set of critical values of $f$ over $\mathbb{C}$. Note that the $z_{i}$ are algebraic over $\mathbb{Q}$; hence, we can choose a nonzero integer $N$ such that the $N z_{i}$ lie in the integral closure of $\mathbb{Z}$ inside $\mathbb{C}$. Write $\mathcal{O}_{z_{i}}$ for the integral closure of $\mathcal{O}\left[N z_{i}\right]$ inside its fraction field. Let $Z_{i}$ be the intersection of $Z$ with the closed subvariety of $\mathbb{A}_{\mathcal{O}_{z_{i}}}^{n}$ given by $N f(x)=N z_{i}$. By [13, Remark 4.5.3] and [5, Lemma 5.1], there is $M$ such that for any local field $L$ over $\mathcal{O}$ of residue field characteristic at least $M$ and any nontrivial additive character $\psi$ on $L$ with $m_{\psi} \geqslant 2$, we have

$$
E_{f, L, \psi}^{Z}=\sum_{i} E_{f, L, \psi}^{Z_{i}}
$$

where the sum is over those $i$ such that $L$ admits a unit-preserving ring homomorphism $\mathcal{O}_{z_{i}} \rightarrow L$. (Here, we use the fact that $m_{\psi} \geqslant 2$.) Up to working with each $Z_{i}$ separately, with $f$ replaced by $N\left(f-z_{i}\right)$, it follows from (3.12) that it is enough to only consider $Z$ such that $f$ vanishes on $Z(\mathbb{C})$. But this case follows by combining the estimates and equalities from Propositions 3.1, 3.4, 3.5, 3.6, and Corollary 2.10. Indeed, there clearly exists a uniform bound on the number of characters of $\mathcal{O}_{L}^{\times}$with order dividing $\prod_{i \in T_{K}} N_{i}$, and, by Propositions 3.1 and 3.4, these characters are the only ones that can contribute to the exponential sums $E_{f, L, \psi}^{Z}$.

By the uniformity in $Z$ in the results used in the proof of Theorem 1.5, we can show a uniform variant, where both constants $C$ and $M$ can be taken independently from $Z$. For simplicity of notation, we focus on the case that $f$ vanishes on $Z(\mathbb{C})$, leaving to the reader the task of formulating and proving the variant of Theorem 3.7 without the condition $f(Z(\mathbb{C}))=0$. Consider a ring of integers $\mathcal{O}_{1}$ containing $\mathcal{O}$ and a closed subscheme $Z$ of $\mathbb{A}_{\mathcal{O}_{1}}^{n}$ with $f(Z(\mathbb{C}))=0$. For such data and any finite field $\mathbb{F}_{q}$ of large enough characteristic (depending only on $f$ and $h$ ) allowing a ring morphism $\mathcal{O}_{1} \rightarrow \mathbb{F}_{q}$, define

$$
\tau_{Z, \mathbb{F}_{q}}(f)=\min _{i} \frac{\nu_{i}}{N_{i}},
$$

where the minimum is taken over those $i \in T_{K}$ such that $h\left(E_{i}\right)\left(\mathbb{F}_{q}\right)$ has nonempty intersection with $Z\left(\mathbb{F}_{q}\right)$ and using notation for the reduction of $h\left(E_{i}\right)$ as before.

THEOREM 3.7 (Uniformity in Z). Let $N>0$ and $n>0$ be integers and let $f \in \mathcal{O}\left[x_{1}, \ldots, x_{n}\right]$ be a nonconstant polynomial over a ring of integers $\mathcal{O}$. Then there exist $C>0$ and $M>0$ such that for each ring of integers $\mathcal{O}_{1}$ containing $\mathcal{O}$, 
for each closed subscheme $Z$ of $\mathbb{A}_{\mathcal{O}_{1}}^{n}$ with $f(Z(\mathbb{C}))=0$ and such that $Z$ is defined by at most $N$ equations, each of degree at most $N$, for each local field $L$ over $\mathcal{O}_{1}$ with residue field characteristic at least $M$, and for each nontrivial additive character $\psi$ on $L$ with $m_{\psi} \geqslant 2$, we have

$$
\left|E_{f, L, \psi}^{Z}\right|<C m_{\psi}^{n-1} q_{L}^{-\tau_{Z, k_{L}}(f) m_{\psi}} .
$$

The case of Theorem 3.7 with the extra assumption that $Z$ is a point in $\mathcal{O}^{n}$ was predicted in [10, Conjecture 1.2 (1.2.2)].

Proof of Theorem 3.7. The proof follows closely the proof of Theorem 1.5 by exploiting in addition the uniformity assertions in Propositions 3.1, 3.4, 3.5, and 3.6.

Note that, given any $\mathcal{O}_{1}$ and $Z$ as in Theorem 3.7, there is $M_{Z}$ such that $\tau_{Z, k_{L}}(f)$ equals $\sigma_{Z}(f)$ for all $L$ over $\mathcal{O}_{1}$ with $p_{L}>M_{Z}$. However, in Theorem 3.7, we can take $M$ independent of $Z$.

3.3. Poles of largest possible order. We formulate a consequence of our work to poles of maximal possible order for Igusa's local zeta functions in the twisted case. Recall that Veys' 1999 conjecture from [31], solved by Nicaise and $\mathrm{Xu}$ in [37], says that any pole of maximal possible order for Igusa's local zeta function associated with $f$ is of the form $-1 / N$, for a positive integer $N$; moreover, in this case, the $\log$ canonical threshold of $f$ is equal to $1 / N$. Recall that for any polynomial $f$ in $n$ variables over $\mathcal{O}$, and closed subscheme $Z$ of $\mathbb{A}_{\mathcal{O}}^{n}$, any local field $L$ over $\mathcal{O}$ of characteristic zero, and any character $\chi$ of $\mathcal{O}_{L}^{\times}$, the maximal possible order of any pole of $\mathcal{Z}_{f, L, \chi, s}^{Z}$ is $n$. Nicaise and Xu treat the nontwisted case of Igusa's local zeta function with $Z=\{0\}$, namely, $\mathcal{Z}_{f, L, \chi_{\text {triv }}, s}$, with $\chi_{\text {triv }}$ being the trivial character.

Our work has consequences for poles of maximal possible order in the twisted case of Igusa's local zeta function around $Z$, namely, for $\mathcal{Z}_{f, L, \chi, s}^{Z}$ with nontrivial $\chi$. It is easy to check that if $s_{0}$ is a pole of maximal possible order of

$$
\mathcal{Z}_{f, L, \chi \text { triv }, s}^{Z}
$$

and if $\chi$ has order $d$, then $s_{0} / d$ is a pole of maximal possible order of

$$
\mathcal{Z}_{f^{d}, L, \chi, s}^{Z} \text {. }
$$

Motivated by this observation and by [31], [37], one may wonder whether $s_{0}$ being a pole of maximal possible order of $\mathcal{Z}_{f, L, \chi, s}^{Z}$ with $\chi$ a character of order $d>1$ implies that $s_{0}=-\operatorname{lct}_{Z}(f)=-1 / d k$ for a positive integer $k$. We obtain the following result in this direction. 
PROPOSITION 3.8. Let $f$ and $Z$ be as above and let $d \geqslant 1$ be an integer. If $s_{0}=-\operatorname{lct}_{Z}(f)$ is a pole of maximal possible order of $\mathcal{Z}_{f, L, \chi, s}^{Z}$ for infinitely many $L$ with arbitrarily large residue field characteristic and with $\chi$ a character of order $d$ on $\mathcal{O}_{L}^{\times}$, then

$$
\operatorname{lct}_{Z}(f) \leqslant \frac{1}{d}
$$

Proof. If $s_{0}=-\operatorname{lct}_{Z}(f)$ is a pole of maximal possible order of $\mathcal{Z}_{f, L, \chi, s}^{Z}$ for infinitely many $L$ with arbitrarily large residue field characteristic, then the power condition holds for $(f, h, Z)$ witnessed by some $(I, W, g, d)$ with $|I|=n$, $d \mid N_{i}$ and $\operatorname{lct}_{Z}(f)=v_{i} / N_{i}$ for all $i \in I$. Indeed, this follows from Proposition 3.4. The proposition now follows by applying the bound (2.8) from Corollary 2.10 for this $I$ and $d$.

3.4. Optimality of the bounds and the motivic oscillation index. In this last section, we give lower bounds for $\left|E_{f, L, \psi}^{Z}\right|$, showing the optimality of the exponent $\sigma_{Z}(f)$ in the bounds of Theorem 1.5 when $\sigma_{Z}(f)<1$ and, in some cases, also when $\sigma_{Z}(f)=1$. In fact, we refine the notion of motivic oscillation index of $f$ from [7] to a variant $\operatorname{moi}_{Z}(f)$ around $Z$ (with a sign change compared to [7]) and show, on one hand, the optimality of bounds with $\operatorname{moi}_{Z}(f)$ in the exponent and, on the other hand, the equality $\operatorname{moi}_{Z}(f)=\sigma_{Z}(f)$ in the case of nonrational singularities. We will conclude by rephrasing the remaining part of Igusa's conjecture optimally in terms of $\operatorname{moi}_{Z}(f)$.

We use the notation in Section 3.1. Thus, $f$ is a nonconstant polynomial in $n$ variables with coefficients in $\mathcal{O}, Z$ is a closed subscheme of $\mathbb{A}_{\mathcal{O}}^{n}$, and $h$ is a log resolution of $D_{K}$, with $K$ the field of fractions of $\mathcal{O}$. Also, in this section, we only work with local fields $L$ which are either of characteristic zero or of any characteristic but with $p_{L}$ sufficiently large (depending on $f$ and $h$ ).

With the notation from Section 3.1 for $\chi$, recall that $\mathcal{Z}_{f, L, \chi, s}^{Z}$ equals a rational function $R(t)$ in $t=q_{L}^{-s}$, and denote by

$$
\mathrm{NP}_{f, L, \chi}^{Z}
$$

the set of complex numbers $s_{0}$ such that $t_{0}:=q_{L}^{-s_{0}}$ is a pole of

$$
\left(t-q_{L}\right)^{\delta} R(t)
$$

with $\delta=1$ if $\chi$ is trivial and with $\delta=0$ if $\chi$ is nontrivial. This is the set of nontrivial poles of $\mathcal{Z}_{f, L, \chi, s}^{Z}$.

Define

$$
\operatorname{LNP}_{f, L, \chi}^{Z}:=\sup \left\{\Re(r) \mid r \in \mathrm{NP}_{f, L, \chi}^{Z}\right\},
$$


where $\mathfrak{R}(r)$ stands for the real part of $r$ and where the supremum over the empty set is taken to be $-\infty$. This is the (real part of the) largest nontrivial pole of $\mathcal{Z}_{f, L, \chi, s}^{Z}$.

If $f$ vanishes on $Z(\mathbb{C})$, then we define the motivic oscillation index of $f$ along $Z$ as

$$
\operatorname{moi}_{Z}(f):=-\lim _{M \rightarrow+\infty} \sup _{L, p_{L}>M} \sup _{\chi}\left(\operatorname{LNP}_{f, L, \chi}^{Z}\right),
$$

where $L$ runs over the local fields over any ring of integers containing the coefficients of $f$ and over which $Z$ can be defined, with residue field characteristic $p_{L}$ larger than $M$ and $\chi$ runs over all multiplicative characters $\mathcal{O}_{L}^{\times} \rightarrow \mathbb{C}^{\times}$. Note that the limit of the suprema in the definition of $\operatorname{moi}_{Z}(f)$ stabilizes; see [7, Corollary 3.4]. A definition of $\operatorname{moi}_{Z}(f)$ for $f$ and $Z$ defined over the algebraic closure of $\mathbf{Q}$ rather than over a ring of integers is given in [9], where also some new inequalities are shown.

For general $Z$, for any critical value $z_{i}$ of $f$, let $Z_{i}$ be the intersection of $Z$ with the closed subvariety of $\mathbb{A}_{\mathcal{O}_{z_{i}}}^{n}$ given by $N f(x)=N z_{i}$ for some nonzero integer $N$ such that the $N z_{i}$ lie in the integral closure of $\mathbb{Z}$ inside $\mathbb{C}$ and with $\mathcal{O}_{z_{i}}$ as in the proof of Theorem 1.5. We define $\operatorname{moi}_{Z}(f)$ as the minimum over $i$ of the values $\operatorname{moi}_{Z_{i}}\left(N\left(f-z_{i}\right)\right)$.

It is a result of Igusa (see [23, Theorem 2]) that $\mathrm{LNP}_{f, L, \chi}^{Z}$ is a negative rational number or $-\infty$. Moreover, if $f(Z(\mathbb{C}))=0$, then $\operatorname{moi}_{Z}(f)$ is either $+\infty$ or a positive rational number equal to $v_{i} / N_{i}$ for some $i \in J$, where we use the notation in Section 2.1.

The following result gives lower bounds for the exponential sums in terms of $\operatorname{moi}_{Z}(f)$ in the exponent. It follows directly from [23, Theorem 2] and the observation at the start of [23, Section 4]. In combination with Proposition 3.10, it shows optimality of our bounds in the case of nonrational singularities around $Z$. Indeed, when $f(Z(\mathbb{C}))=0$, Proposition 3.10 shows that $\operatorname{lct}_{Z}(f)=\operatorname{moi}_{Z}(f)$ if and only if $f$ has nonrational singularities on every open neighborhood $V$ of $Z_{\mathbb{C}}$ in $\mathbb{A}_{\mathbb{C}}^{n}$.

Proposition 3.9 [23]. Given $f$ and $Z$ as above, there exist infinitely many local fields $L$ over $\mathcal{O}$ (with arbitrarily large residue field characteristic), constants $c_{L}>0$, and positive integers $a$, $c$, such that

$$
c_{L} q_{L}^{-\operatorname{moi}_{Z}(f) m} \leqslant\left|E_{f, L, \psi}^{Z}\right|
$$

for each $m \in c+a \mathbb{N}$ and some additive character $\psi$ on $L$ with $m_{\psi}=m$.

Proof. The assertion follows from [23, Theorem 2] (see also [13, Corollary 1.4.5] and the comment to [13] at the end of [16]). 
Proposition 3.10. Given $f$ and $Z$ as above, there exists an open neighborhood $V$ of $Z_{\mathbb{C}}$ in $\mathbb{A}_{\mathbb{C}}^{n}$ such that the following equivalences hold. All hypersurfaces defined by $f-b$, for $b \in \mathbb{C}$, have rational singularities on $V$ if and only if $\operatorname{moi}_{Z}(f)>1$. If furthermore $f$ vanishes on $Z(\mathbb{C})$, then we have $\operatorname{moi}_{Z}(f) \leqslant 1$ if and only if $\operatorname{moi}_{Z}(f)=\operatorname{lct}_{Z}(f)$.

Proof. The proof relies mainly on [23, Section 3], using the fact that the observation at the beginning of [23, Section 4$]$ removes the condition that 0 is the only critical value. Clearly, it is enough to consider the case when $f(Z(\mathbb{C}))=0$. In what follows, we use the notation in Section 2.1 for a $\log$ resolution $h$. If $f$ has no singularities on some $V$ containing $Z$, then $\operatorname{moi}_{Z}(f)=+\infty$ since then the sets $\mathrm{NP}_{f, L, \chi}^{Z}$ are empty whenever $p_{L}$ is large.

Note that the hypersurface defined by $f$ has rational singularities on some $V$ containing $Z_{\mathbb{C}}$ if and only if for each $i \in J$ with $h\left(E_{i}\right) \cap Z$ nonempty either $v_{i} / N_{i}>1$ or $\left(N_{i}, v_{i}\right)=(1,1)$ and for every other $E_{i^{\prime}}$ with $\left(N_{i^{\prime}}, v_{i^{\prime}}\right)=(1$, 1 ), we have $E_{i} \cap E_{i^{\prime}} \cap h^{-1}(Z)=\emptyset$ (indeed, the latter condition is known as the pair $\left(\mathbb{A}_{\mathbb{C}}^{n}, D\right)$ having canonical singularities in a neighborhood of $Z_{\mathbb{C}}$; this is equivalent with $D$ having rational singularities in a neighborhood of $Z_{\mathbb{C}}$ by [29, Theorems 7.9 and 11.1]). If these properties on the numerical data hold, then [23, Theorem 2] implies that $\operatorname{LNP}_{f, L, \chi}^{Z}<-1$ for all $L$ and all $\chi$ whenever $p_{L}$ is large and, hence, that $\operatorname{moi}_{Z}(f)>1$.

Conversely, suppose that $\operatorname{moi}_{Z}(f)>1$. We need to find an open neighborhood $V$ of $Z_{\mathbb{C}}$ such that the hypersurface defined by $f$ has rational singularities in $V$. For this, we follow an argument already present in Igusa's work. Since $\operatorname{moi}_{Z}(f)>1$, it follows from [23, Theorem 2] that for $p_{L}$ large, the function $L^{\vee} \rightarrow \mathbb{C}$ sending a character $\psi$ in the (topological) dual $L^{\vee}$ of $(L,+)$ to the complex number $E_{f, L, \psi}^{Z}$ is an $\mathrm{L}^{1}$-function (with respect to the Haar measure). By [23, Theorem 2], for any $L$, this function $\psi \mapsto E_{f, L, \psi}^{Z}$ is $L^{1}$ on $L^{\vee}$ if and only if the limit of

$$
F_{L}^{Z}(k):=\int_{\left\{x \in \mathcal{O}_{L}^{n} \mid \bar{x} \in Z\left(k_{L}\right), f(x)=k\right\}}|d x / d f|
$$

exists for $k \rightarrow 0$, where $|d x / d f|$ stands for the volume associated with the Gelfand-Leray differential form on $f(x)=k$ for smooth values $k \in L$ of $f$. By [23, Lemma 4], we can find $V$ as needed if and only if for all $L$ with $p_{L}$ sufficiently large and all real-valued, nonnegative Schwartz-Bruhat functions $\Phi$ on $L^{n}$ such that $f(\operatorname{Supp} \Phi)$ contains no critical value of $f$ other than 0 , where Supp $\Phi$ is the support of $\Phi$, we have that the limit of

$$
F_{\Phi}(k):=\int_{\left\{x \in L^{n} \mid f(x)=k\right\}} \Phi(x)|d x / d f|
$$


for $k \rightarrow 0$ exists. Note that $F_{\Phi}$ is nonnegatively real-valued since $\Phi$ is. Moreover, it follows from [23, Theorem 2] that if the limit of $F_{\Phi}$ for $k \rightarrow 0$ does not exist, then for each $C$, there is $k \in L^{\times}$with

$$
C<F_{\Phi}(k)
$$

(that is, arbitrarily large values for $F_{\Phi}$ occur in this situation). Note also that if $f(\operatorname{Supp} \Phi)$ contains no critical value of $f$, then $F_{\Phi}$ is continuous on $L$. From this discussion and the additivity of $F_{\Phi}$ in nonnegative real-valued $\Phi$, it follows that we can find $V$ as desired, namely, an open neighborhood of $Z_{\mathbb{C}}$ in $\mathbb{A}_{\mathbb{C}}^{n}$ such that the hypersurface defined by $f$ has rational singularities on $V$. (Alternatively, one can use [1, Theorem 3.4] and the equivalence between its statements a and $\mathrm{c}$ to shorten the above argument.)

Let us now prove that $\operatorname{moi}_{Z}(f) \leqslant 1$ if and only if $\operatorname{moi}_{Z}(f)=\operatorname{lct}_{Z}(f)$. This follows again by an argument already present in Igusa's work. The fact that if $\operatorname{moi}_{Z}(f)=\operatorname{lct}_{Z}(f)$, then $\operatorname{moi}_{Z}(f) \leqslant 1$ is clear since we always have $\operatorname{lct}_{Z}(f) \leqslant 1$. Suppose now that $\operatorname{moi}_{Z}(f) \leqslant 1$. As soon as $L$ contains a large enough finite field extension of the field of fractions $K$ of $\mathcal{O}$, we have that $s=-\operatorname{lct}_{Z}(f)$ is a pole of $\mathcal{Z}_{f, L, \chi_{\text {triv }, s}}^{Z}$ by the proof of [23, Lemma 4] (alternatively, and with more details, by [41, Theorem 2.7]). We always have $\operatorname{moi}_{Z}(f) \geqslant \operatorname{lct}_{Z}(f)$ since $\operatorname{moi}_{Z}(f)$ is either $+\infty$ or $v_{i} / N_{i}$ for some $i \in J$. If $\operatorname{lct}_{Z}(f)<1$, then we are done since then clearly $-\operatorname{lct}_{Z}(f)$ is also a pole of $\left(t-q_{L}\right) R(t)$, where $R\left(q_{L}^{-s}\right)=\mathcal{Z}_{f, L, \chi_{\text {triv }}, s}$. On the other hand, if $\operatorname{lct}_{Z}(f)=1$, then we are done since $\operatorname{moi}_{Z}(f) \leqslant 1$ and $\operatorname{moi}_{Z}(f) \geqslant \operatorname{lct}_{Z}(f)$.

Note that $\operatorname{moi}_{Z}(f)$ always gives upper bounds with constants $c_{L}$ depending on $L$ by Igusa's work [21] and [23] (see also [13, Corollary 1.4.5] and [16]), as follows.

Proposition 3.11 (Igusa). Given $f$ and $Z$, there exist $M$, and for each $L$ over $\mathcal{O}$ with $p_{L}>M$, a constant $c_{L}>0$ such that for all nontrivial additive characters $\psi$ on $L$ with $m_{\psi} \geqslant 2$, we have

$$
\left|E_{f, L, \psi}^{Z}\right| \leqslant c_{L} q_{L}^{-\operatorname{moi}_{Z}(f) m_{\psi}} m_{\psi}^{n-1} .
$$

Note that the exponent $n-1$ of $m_{\psi}$ in (3.15) is not always optimal and is related to the order of the largest nontrivial pole of Igusa's zeta functions; one can define naturally a multiplicity of the motivic oscillation index capturing the optimal exponent of $m_{\psi}$. Let us finally recall the strong form of Igusa's conjecture with the motivic oscillation index around $Z$, predicting that the constants $c_{L}$ in (3.15) can be taken independently from $L$ as soon as $p_{L}$ is large enough. By the work of this paper, this remains open in general only if $\operatorname{moi}_{Z}(f)>1$. 
Let us finally note that in the last chapter of Nguyen's PhD thesis [36], it was shown that the inequalities from (2.9) and Theorem 1.5 are equivalent (without proving either in general). The results from the thesis also inspired $\mathrm{W}$. Veys to consider the inequality from (2.9) in the 2-variable case; see [40].

\section{Acknowledgements}

The authors R.C. and K.H.N. are partially supported by the European Research Council under the European Community's Seventh Framework Programme (FP7/2007-2013) with ERC Grant Agreement number 615722 MOTMELSUM, by the Labex CEMPI (ANR-11-LABX-0007-01) and by KU Leuven IF C14/17/083. K.H.N. is partially supported by Fund for Scientific Research - Flanders (Belgium) (F.W.O.) 12X3519N. M.M. is partially supported by NSF grant DMS-1701622. The authors would like to thank Ben Lichtin, Andrei Mustaţă, Johannes Nicaise, Antonio Rojas-León, and Wim Veys for advice and interesting discussions on the topics of this paper and the referee for valuable comments. The authors are also grateful to Jan Denef for his long-term guidance in this subject.

\section{References}

[1] A. Aizenbud and N. Avni, 'Representation growth and rational singularities of the moduli space of local systems', Invent. Math. 204 (2016), 245-316.

[2] C. Birkar, P. Cascini, C. D. Hacon and J. McKernan, 'Existence of minimal models for varieties of log general type', J. Amer. Math. Soc. 23 (2010), 405-468.

[3] H. Blum, 'On divisors computing mld's and lct's'. Preprint, 2016, arXiv:1605.09662.

[4] W. Castryck and K. H. Nguyen, 'New bounds for exponential sums with a non-degenerate phase polynomial', J. Math. Pures Appl. (9) (2019), doi:10.1016/j.matpur.2019.01.015.

[5] S. Chambille and K. H. Nguyen, Proof of Cluckers-Veys's conjecture on exponential sums for polynomials with log canonical threshold at most a half, Int. Math. Res. Not. IMRN 2019, rnz036, doi:10.1093/imrn/rnz036.

[6] R. Cluckers, 'Igusa and Denef-Sperber conjectures on nondegenerate $p$-adic exponential sums', Duke Math. J. 141 (2008), 205-216.

[7] R. Cluckers, 'Igusa's Conjecture on exponential sums modulo $p$ and $p^{2}$ and the motivic oscillation index', Int. Math. Res. Not. IMRN (4) (2008), article ID rnm118, 20 pages.

[8] R. Cluckers, 'Exponential sums: questions by Denef, Sperber, and Igusa', Trans. Amer. Math. Soc. 362 (2010), 3745-3756.

[9] R. Cluckers and M. Mustaţă, 'An invariant detecting rational singularities via the log canonical threshold'. Preprint, 2019, arXiv:1901.08111.

[10] R. Cluckers and W. Veys, 'Bounds for log-canonical thresholds and $p$-adic exponential sums', Amer. J. Math. 138 (2016), 61-80.

[11] T. Cochrane, 'Bounds on complete exponential sums', in Analytic Number Theory, Vol. 1 (Allerton Park, IL, 1995), Progress in Mathematics, 138 (Birkhäuser Boston, Boston, MA, 1996), 211-224.

[12] J. Denef, 'Local zeta functions and Euler characteristics', Duke Math. J. 63 (1991), 713-721. 
[13] J. Denef, 'Report on Igusa's local zeta function, Séminaire Bourbaki, Vol. 1990/91', Astérisque 201-203 (1991), Exp. No. 741, 359-386 (1992).

[14] J. Denef and K. Hoornaert, 'Newton polyhedra and Igusa's local zeta function', J. Number Theory 89 (2001), 31-64.

[15] J. Denef and S. Sperber, 'Exponential sums mod $p^{n}$ and Newton polyhedra', Bull. Belg. Math. Soc. Simon Stevin 2001 55-63. suppl.

[16] J. Denef and W. Veys, 'On the holomorphy conjecture for Igusa's local zeta function', Proc. Amer. Math. Soc. 123 (1995), 2981-2988.

[17] H. Hironaka, 'Resolution of singularities of an algebraic variety over a field of characteristic zero. I', Ann. of Math. 79(2) (1964), 109-203.

[18] K. Hoornaert, 'Newton polyhedra, unstable faces and the poles of Igusa's local zeta function', Trans. Amer. Math. Soc. 356 (2004), 1751-1779.

[19] J. Igusa, 'On certain representations of semi-simple algebraic groups and the arithmetic of the corresponding invariants. I', Invent. Math. 12 (1971), 62-94.

[20] J. Igusa, 'On the arithmetic of Pfaffians', Nagoya Math. J. 47 (1972), 169-198.

[21] J. Igusa, 'Complex powers and asymptotic expansions I', J. Reine Angew. Math. 268/269 (1974), 110-130.

[22] J. Igusa, 'On a certain Poisson formula', Nagoya Math. J. 53 (1974), 211-233.

[23] J. Igusa, 'Complex powers and asymptotic expansions II', J. Reine Angew. Math. 278/279 (1975), 307-321.

[24] J. Igusa, 'A Poisson formula and exponential sums', J. Fac. Sci. Univ. Tokyo Sect. IAMath. 23 (1976), 223-244.

[25] J. Igusa, 'Criteria for the validity of a certain Poisson formula', in Algebraic Number Theory (Kyoto Internat. Sympos., Res. Inst. Math. Sci., Univ. Kyoto, Kyoto, 1976) (Japan Soc. Promotion Sci., Tokyo, 1977), 43-65.

[26] J. Igusa, Lectures on Forms of Higher Degree (notes by S. Raghavan), Lectures on Mathematics and Physics, 59 (Tata institute of fundamental research, Springer-Verlag, 1978).

[27] J. Igusa, An Introduction to the Theory of Local Zeta Functions, AMS/IP Studies in Advanced Mathematics, 14 (American Mathematical Society, Providence, RI, 2000), International Press, Cambridge, MA.

[28] N. Katz, 'Sums of Betti numbers in arbitrary characteristic', Finite Fields Appl. 7 (2001), 29-44.

[29] J. Kollár, 'Singularities of pairs', in Algebraic Geometry-Santa Cruz 1995, Proceedings of Symposia in Pure Mathematics, 62 (American Mathematical Society, Providence, RI, 1997), 221-287.

[30] J. Kollár and S. Mori, Birational Geometry of Algebraic Varieties, Cambridge Tracts in Mathematics, 134 With the collaboration of C. H. Clemens and A. Corti, Translated from the 1998 Japanese original, Cambridge University Press, Cambridge, 1998.

[31] A. Laeremans and W. Veys, 'On the poles of maximal order of the topological zeta function', Bull. Lond. Math. Soc. 31 (1999), 441-449.

[32] B. Lichtin, 'On a conjecture of Igusa', Mathematika 59 (2013), 399-425.

[33] B. Lichtin, 'On a conjecture of Igusa II', Amer. J. Math. 138 (2016), 201-249.

[34] H. Matsumura, Commutative Ring Theory, 2nd edn, Cambridge Studies in Advanced Mathematics, 8 Translated from the Japanese by M. Reid, Cambridge University Press, Cambridge, 1989.

[35] M. Mustaţă, 'IMPANGA lecture notes on log canonical thresholds (notes by Tomasz Szemberg)', in Contributions to Algebraic Geometry, EMS Series of Congress Reports (European Mathematical Society, Zürich, 2012), 407-442. 
[36] K. H. Nguyen, 'Uniform rationality of Poincaré series of $p$-adic equivalence relations and Igusa's conjecture on exponential sums', PhD thesis, University of Lille (2018), arXiv:1903.06738.

[37] J. Nicaise and C. Xu, 'Poles of maximal order of motivic zeta functions', Duke Math. J. 165 (2016), 217-243.

[38] A. Rojas-León, 'Estimates for singular multiplicative character sums', Int. Math. Res. Not. IMRN 20 (2005), 1221-1234.

[39] M. Saito, 'On b-function, spectrum and rational singularity', Math. Ann. 295 (1993), 51-74.

[40] W. Veys, 'On the log canonical threshold and numerical data of a resolution in dimension 2'. Preprint, 2018, arXiv:1810.11062.

[41] W. Veys and W. A. Zúñiga-Galindo, 'Zeta functions for analytic mappings, logprincipalization of ideals, and Newton polyhedra', Trans. Amer. Math. Soc. 360 (2008), 2205-2227.

[42] J. Wright, 'Exponential sums and polynomial congruences in two variables: the quasihomogeneous case', Preprint, 2012, arXiv:1202.2686v1. 